RAE-IC, Revista de la Asociación Española de Investigación de la Comunicación vol. 8, núm. 15 (2021), 292-314 ISSN 2341-2690

Recibido el 30 de noviembre de 2020 DOI: https://doi.org/10.24137/raeic.8.15.24 Aceptado el 27 de diciembre de 2020

\title{
La televisión como herramienta para la integración social: narraciones y experiencias de migrantes asiáticos en la ciudad de Mesina (Italia)
}

\author{
Television as a tool for social integration: discourses and experiences of \\ Asian migrants in the city of Messina (Italy)
}

\author{
Olmos Alcaraz, Antonia \\ Universidad de Granada (UGR) \\ antonia@ugr.es \\ Cava, Gaetana \\ Universidad de Granada (UGR) \\ gaetanacava@correo.ug.es
}

Forma de citar este artículo:

Olmos Alcaraz, A. y Cava, G. (2021). La televisión como herramienta para la integración social: narraciones y experiencias de migrantes asiáticos en la ciudad de Mesina (Italia). RAE-IC, Revista de la Asociación Española de Investigación de la Comunicación, 8(15), 292-314. https://doi.org/10.24137/raeic.8.15.24

\section{Resumen:}

El trabajo analiza el uso y consumo que una muestra de personas migrantes asiáticas residentes en Italia hacen de la televisión, con el objetivo de conocer las potencialidades de este medio de comunicación en tanto que herramienta para integración social. Para 
ello se ha trabajado a través de una entrevista semiestructurada, aplicada a 60 personas originarias de China, Filipinas y Sri Lanka, vecinos de la ciudad de Mesina. Los resultados de la investigación indican que las personas participantes en el estudio aprovechan ciertos contenidos y formatos que proporciona la televisión para reforzar el aprendizaje de la lengua y el conocimiento de la sociedad italiana (elementos esenciales dentro de los procesos de integración). Concluimos por ello que la televisión cuenta con potencial para ser un buen recurso para la integración social, sin que ello obvie la necesidad de no realizar análisis simplistas y superficiales que ignoren el carácter procesual, multidimensional y multidireccional de dichos procesos de integración.

Palabras clave: migrantes asiáticos, televisión, integración social, lengua, cultura.

\section{Abstract:}

The paper analyses the use and consumption that a sample of Asian migrants living in Italy make of television, with the aim of knowing the potential of this media as a tool for social integration. For this, we have worked through a semi-structured interview, applied to 60 people from China, the Philippines and Sri Lanka, resident in the city of Messina. The results of the research indicate that the people participating in the study take advantage of certain contents and formats provided by television to reinforce language learning and knowledge of Italian society (essential elements within integration processes). We therefore conclude that television has the potential to be a good resource for social integration, without this obviating the need not to carry out simplistic and superficial analysis that ignore the multidimensional and multidirectional nature of the process of the integration.

Keywords: Asian migrants, television, social integration, language, culture.

\section{INTRODUCCIÓN}

El fenómeno migratorio en Italia presenta a día de hoy características que nos permiten hablar de un arraigo creciente de las personas migrantes (Cáritas, 2012), a pesar de 
existencia en la actualidad de opiniones y discursos que apuntan a todo lo contrario. Esto último -al igual que en otros contextos del entorno-, casi siempre procede de la extrema derecha (Kaya, 2017; Osmani, 2019).

Con respecto a los flujos migratorios, como sucede en otros países de Europa con similar tradición migratoria -por ejemplo España o Portugal-, han disminuido ligeramente en el contexto de crisis. Pero ello es así con respecto a las entradas por motivos labores, sucediendo lo contrario en relación a las personas que llegan al país gracias a las reunificaciones familiares. Esta realidad incide de lleno en las dinámicas de cambio social acontecidas en el territorio, en diversos ámbitos, entre ellos el económico y el cultural; y por supuesto también con respecto a la población autóctona (Meli, 2011). La importancia de las migraciones en tanto que fenómeno a tener en cuenta para conocer sobre las transformaciones culturales en el país se puso de manifiesto de manera muy clara en el año 2013, cuando Aditel decide incluir a 400 familias migrantes en sus estudios sobre audiencia televisiva. En ello puede verse un reconocimiento de la relevancia de esta población para la elaboración de la programación y también en términos de inversión publicitaria (Musarò y Parmiggiani, 2014).

En este sentido este estudio trata de ser una contribución a la investigación sobre las transformaciones culturales relacionadas con el fenómeno migratorio, reflexionado para ello sobre el uso que las personas migrantes en el país hacen de los medios de comunicación (a partir de un estudio de caso en la ciudad de Mesina); y para ello pretendemos visibilizar las narraciones de las propias personas migrantes con respecto al consumo mediático, en concreto, analizando el uso que que hacen de la televisión en tanto que posible herramienta de integración.

\subsection{EL FENÓMENO MIGRATORIO EN LA CIUDAD DE MESINA}

En esta investigación se ha optado por un diseño metodológico de carácter cualitativomicro, razón por la que se decide acotar el contexto de estudio a un territorio: Mesina. Esta ciudad, situada al sur de Italia, es de tamaño medio, contando con 245.260 habitantes según datos del último censo nacional (2011). En el caso de los residentes procedentes de las migraciones extranjeras se registra un aumento significativo en las 
últimas 2 décadas, contando con 4.789 personas de nacionalidad extranjera en 2001, dato que asciende hasta 11.777 residentes una década después, y que se sitúa en $12.478^{1}$ a día de hoy. En relación a los lugares de procedencia decir que la mayoría de estas personas proceden de Asia (casi un $57 \%$ de las mismas), y además casi la totalidad de estas (más del 97\%) son de Sri Lanka, Filipinas o China (Salanitro, 2012).

\subsection{LA INVESTIGACIÓN SOBRE MEDIOS DE COMUNICACIÓN Y EL FENÓMENO MIGRATORIO}

La investigación dirigida a analizar las relaciones entre medios de comunicación y el fenómeno migratorio se ha enfocado en primera instancia, muy especialmente, en el estudio de las representaciones de dichas poblaciones en distintos medios de comunicación. En esta línea se sitúan los trabajos de Balma (2001), Binotto, Bruno y Lai (2016), Bañón (2002, 2007), Calvanese (2011), García, Granados, Olmos Alcaraz y Martínez (2014), Granados (1998, 2013), King y Wood (2013), Lorite (2004a, 2004b, 2011), Martínez (2013, 2015), Martínez y Olmos Alcaraz (2015), Olmos Alcaraz (2013), Retis (2004, 2013) o Van Dijk (1997, 2009). Pese a determinadas particularidades en los resultados de dichas investigaciones, de las mismas se extrae la existencia de una representación en esencia negativa y problematizada del fenómeno de las migraciones en los distintos medios de comunicación y contextos considerados.

En un segundo momento, un poco más adelante en el tiempo, encontramos cómo también se ha avanzado -aunque muy tímidamente-en el análisis de espacios, soportes o plataformas "dirigidas a" o "gestionados por" la propia población migrante. Entre este tipo de trabajos destacan las investigaciones sobre los conocidos como ethnic media (Matsaganis, Katz y Ball, 2010). Algunos ejemplos de trabajos a este respecto son los de Santillán (2008) sobre los medios dirigidos a la comunidad latina en España; los trabajos de Navarro (2014, 2015) sobre medios de comunicación creados por migrantes marroquíes en España (2014) y sobre los programas de la televisión pública española dirigidos a la población procedente de las migraciones (2015); o los trabajos de Rossel

\footnotetext{
${ }^{1}$ Datos ofrecidos por el Ayuntamiento de Mesina. Ver https://www.tuttitalia.it/sicilia/38-messina/statistiche/cittadini-stranieri2019/
} 
(2008) y González (2009) sobre la profesionalización y consolidación de la prensa dirigida a personas migrantes $y / o$ extranjeras. A este respecto señalamos también una investigación propia sobre el programa Babel TV (Canal Sky, creado en 2010 y emitido hasta 2014), espacio televisivo destinado a la población inmigrante (Cava, 2019).

Por último señalamos otro campo de estudio que relaciona el fenómeno migratorio con los medios de comunicación, en este caso trabajos dedicados a conocer cómo las personas migrantes usan los medios y/o programas generalistas, no dirigidos a ningún segmento poblacional en concreto. No se trata esta, al igual que la anterior, de una línea de investigación hegemónica. Aun así encontramos trabajos interesantes dentro de misma, como los realizados Altarriba (2008) sobre el uso y las actitudes de los migrantes ante los medios de comunicación; el de Belluati (2015) sobre las preferencias y gustos televisivos de esta población; el trabajo de Chavero y García (2005) sobre los hábitos de consumo también televisivo, en este caso, de jóvenes latinoamericanos; y los trabajos realizados por Retis $(2014,2018)$ sobre el surgimiento de audiencias transnacionales, el tipo de medios que consumen y el eco que se hacen de las mismas las agencias publicitarias.

El presente trabajo se sitúa entre los últimos mencionados, y en el mismo tratamos de reconstruir las situaciones concretas del uso de la televisión por parte de un grupo de personas migrantes de procedencia asiática residentes en la ciudad de Mesina. A este respecto nos hacemos eco también de las teorías de usos y gratificaciones (Katz, Blumler y Gurevitch, 1974), muy relacionadas con los estudios sobre audiencias. Dado que dicho enfoque profundiza sobre qué pueden hacer las personas con los medios, incidiendo en el carácter activo y no pasivo de estas en el consumo cultural (Livolsi, 2020), nos interesa especialmente pensar nuestros análisis a la luz de las mismas. Uno de los objetivos de dicho enfoque es conocer las razones por las que las personas usan los mass-media y para qué lo hacen, por lo que suponen una "herramienta teórica fundamental para analizar cómo, cuándo y por qué las personas pasan tiempo con los contenidos de los medios" (Igartúa, Ortega-Mohedano y Arcila-Calderón, 2020, p. 3). En relación a su aplicación para conocer sobre cómo se relacionan las personas migrantes con la televisión (cómo seleccionan contenidos y los utilizan para gratificar sus necesidades y 
voluntades), nuestra investigación recoge las narraciones que los/as propios/as migrantes tienen sobre cómo usan, interpretan y hablan de los espacios que ven; lo que nos sirve para reflexionar sobre el consumo televisivo como posible herramienta para la integración.

\section{DISEÑO METODOLÓGICO}

El objetivo de este trabajo ha sido conocer las pautas de consumo televisivo de las personas migrantes de procedencia asiática en la ciudad de Mesina (Italia), para indagar en los usos dados a la misma en tanto que posible herramienta para la integración.

\subsection{MUESTRA}

Para ello se trabajó a través de la realización de entrevistas semiestructuradas, aplicadas a una muestra (muestreo intencional no estratificado) de 60 personas $(N=60)$. El tipo y diseño de la muestra responde a los objetivos teóricos de la investigación, por lo que los resultados obtenidos no pueden ser extrapolables a toda la población. Con respecto a la composición socio-demográfica y las características de la muestra decir que se contó con 30 hombres y 30 mujeres, entre 30 y 50 años $(M=41)$ y originarios en igual proporción de China (20), Filipinas (20) y Sri Lanka (20), las tres procedencias más numerosas entre la población migrante asiática de la ciudad de Mesina. En cuanto al nivel del estudios el $50 \%$ de la muestra contaban con formación media-superior, el $26,7 \%$ con estudios universitarios, el $13,3 \%$ contaba con formación media-inferior, el 8,3\% tenía formación de máster y/o doctorado y menos de un $2 \%$ manifestó contar con estudios elementales. Más del $90 \%$ de la muestra (91,7\%) se encontraba en activo en el momento de participar en la investigación. El resto indicaron trabajar en las labores del hogar.

\subsection{INSTRUMENTO DE PRODUCCIÓN DE DATOS}

El guion de la entrevista contó con tres grandes bloques, los dos segundos -a su vezdivididos en dos secciones cada uno de ellos. El primer bloque estaba dedicado a conocer los perfiles socio-demográficos de las personas participantes en la investigación. El segundo bloque se reservó para conocer sobre el proceso migratorio 
de los sujetos, con una primera sección enfocada a cuestiones relacionadas con las razones de la migración, la elección del lugar de residencia, el tiempo de estancia, etc.; y la segunda dedicada a elementos culturales y lingüísticos (adquisición, uso, etc.). El tercer bloque sirvió para indagar específicamente en el uso y el consumo televisivo. La primera sección del mismo, con preguntas de carácter más descriptivo, para conocer las pautas y experiencias de dicho uso y consumo (tiempos, espacios, elecciones televisivas, etc.); y la segunda sección, se diseñó para profundizar sobre los significados otorgados a dicho uso y consumo mediático, focalizando en las representaciones sobre la inmigración y los procesos de discriminación e integración en la televisión. Para el presente artículo nos centramos en el análisis de diversos ítems del segundo y tercer bloque, en concreto, relacionados con el uso de la televisión como herramienta para la adquisición y mejora de la lengua y para el conocimiento de elementos culturales del país de acogida.

\subsection{TRATAMIENTO Y ANÁLISIS}

La entrevista se diseñó con un lenguaje asequible y se contó con asesoramiento de traducción en los casos en los que fue necesario. Se aplicó en los domicilios de las personas participantes. Se accedió a las personas a través de la técnica bola de nieve. El tratamiento y análisis de los datos producidos a partir de la entrevista (con preguntas de respuesta cerrada y con preguntas de respuesta libre) se realizó con diversos programas y aplicaciones informáticas. Se trabajó con Google Form para el análisis de frecuencias, con Excel para el análisis de contingencias y con N.Vivo para el análisis de contenido.

\section{RESULTADOS}

En el marco de la investigación nos interesamos por conocer las opiniones y experiencias de las personas migrantes sobre las posibilidades que podría ofrecer la TV como soporte para apoyar algunos elementos centrales en los procesos de integración social: la lengua y la cultura. Para indagar en esta cuestión nos preguntamos: 1). Qué tipo de televisión ven los/as migrantes (la televisión del país de acogida o la televisión de sus países de origen); 2). Si ver la televisión del país de acogida favorece o no (y en qué sentido) la 
mejora de la competencia lingüística; y 3). Si es posible que la televisión funcione como un recurso para conocer la cultura y las costumbres de su actual país de residencia. Pero antes hubo que indagar en la centralidad de este medio de comunicación entre los sujetos que conformaban la muestra.

\subsection{EL CONSUMO TELEVISIVO EN LA COMUNIDAD DE MIGRANTES ASIÁTICOS DE} MESINA

En primera instancia se preguntó por los hábitos de consumo televisivo. El objetivo fue hacernos una idea de la centralidad o falta de la misma de este medio de comunicación entre las personas migrantes participantes en la investigación, para -posteriormenteseguir indagando sobre el uso de la misma como recurso para la integración (en relación al aprendizaje de la lengua y el conocimiento de los códigos culturales italianos).

Como resultado obtuvimos que un escaso $13 \%$ afirmó no ver la TV y hasta un $87 \%$ de la muestra respondió de manera positiva a la pregunta. A este respecto, hay más mujeres que hombres que afirmaron ver la televisión (90\% frente a 83,3\%); no se encontraron diferencias significativas al respecto en función de la edad o la situación laboral²; en relación a la formación se encontraron porcentajes relativamente similares en todos los grupos, a excepción de las personas con educación media-superior que fueron las que en mayor medida respondieron ver televisión (hasta un 95\% de ellos/as, frente a un 80$90 \%$ en el resto de grupos ${ }^{3}$ ); y, por último, con respecto a las procedencias, quienes en mayor medida manifestaron ver la televisión fueron las personas originarias de Sri Lanka (el $95 \%$ de ellos/as), seguidas de las personas procedentes de Filipinas (90\%) y por último quienes procedían de China (75\%).

Entre quienes dieron una respuesta negativa (apenas el 13\% la muestra), las razones esbozadas más frecuentemente estaban relacionadas con el trabajo (no tener tiempo para ello) y con la preferencia de otros medios de comunicación más adaptables a los tiempos y preferencias individuales (ver series o películas en internet).

\footnotetext{
${ }^{2}$ Recuérdese que más del $90 \%$ de la muestra se encontraba en activo laboralmente.

${ }^{3}$ Excluimos del análisis el grupo de personas con "estudios elementales", porque solo había una persona con esta formación y respondió no ver la televisión.
} 


\subsection{LA TELEVISIÓN COMO SOPORTE DE LA INTEGRACIÓN SOCIAL}

Para seguir avanzando en el conocimiento sobre el uso de la televisión como herramienta para la integración social preguntamos si su consumo televisivo era de la televisión nacional o también con respecto a la TV de otros países (Tabla 1). La pregunta, de respuesta cerrada con opción múltiple, se formuló de la siguiente manera: ¿Sueles ver la televisión italiana o la de otros países? ${ }^{4}$ Respondieron a la misma 53 personas (el $88,3 \%$ de la muestra), con los siguientes resultados: la mayoría de las personas, más de la mitad de la muestra $(58,5 \%)$, manifestaron consumir tanto la televisión italiana como la televisión de otros países; casi un 40\% respondieron ver en mayor medida la televisión italiana; y apenas un 2\% indicó normalmente ver solo televisión no italiana.

Con respecto a las diferencias encontradas por género puede verse la siguiente tabla:

Tabla 1. Consumo de televisión (italiana, de otros países o ambas) según género

\begin{tabular}{|c|c|c|c|c|}
\hline \multirow{2}{*}{ Género } & \multicolumn{4}{|c|}{ ¿Sueles ver la televisión italiana o la de otros países? } \\
\cline { 2 - 5 } & Ambas & $\begin{array}{c}\text { La televisión de } \\
\text { otros países }\end{array}$ & $\begin{array}{c}\text { La televisión } \\
\text { italiana }\end{array}$ & Total \\
\hline F & $14(50 \%)$ & $1(3,57 \%)$ & $13(46,42 \%)$ & $28(100 \%)$ \\
\hline M & $17(68 \%)$ & & $8(32 \%)$ & $25(100 \%)$ \\
\hline Total & $31(58,49 \%)$ & $1(1,88 \%)$ & $21(39,62 \%)$ & $53(100 \%)$ \\
\hline
\end{tabular}

Fuente: elaboración propia

Encontramos que el escaso $2 \%$ de la muestra que indica ver solo la televisión de otros países corresponde a una persona de género femenino; tanto mujeres como hombres indican en mayor medida ver ambas televisiones; y con respecto a quienes consumen solo televisión italiana destacan las mujeres.

En cuanto a la edad (Tabla 2), en todas las franjas es más frecuente haber respondido ver ambos tipos de televisión, aunque las diferencias son más grandes entre las personas de más edad (de 41 a 50 años). En otras palabras, mientras que las personas más jóvenes manifestaron en un 53,6\% preferir ver ambas televisiones y en un 42,9\% ver la televisión italiana; el $64 \%$ de las personas de más edad indicaron ver ambas, frente al $36 \%$ de las mismas que respondieron ver solo la televisión italiana:

\footnotetext{
${ }^{4}$ Normalmente vedi la televisione italiana o quella di altri paesi?
} 
Tabla 2. Consumo de televisión (italiana, de otros países o ambas) según edad

\begin{tabular}{|c|c|c|c|c|}
\hline \multirow{2}{*}{ Edad } & \multicolumn{3}{|c|}{ ¿Sueles ver la televisión italiana o la de otros países? } \\
\cline { 2 - 5 } & Ambas & $\begin{array}{c}\text { La televisión de } \\
\text { otros países }\end{array}$ & $\begin{array}{c}\text { La televisión } \\
\text { italiana }\end{array}$ & \multirow{2}{*}{ Total } \\
\hline $\mathbf{3 0 - 4 0}$ & $15(53,57 \%)$ & $1(3,57 \%)$ & $12(42,85 \%)$ & $28(100 \%)$ \\
\hline $\mathbf{4 1 - 5 0}$ & $16(64 \%)$ & & $9(36 \%)$ & $25(100 \%)$ \\
\hline Total & $31(58,49 \%)$ & $1(1,88 \%)$ & $21(39,62 \%)$ & $53(100 \%)$ \\
\hline
\end{tabular}

Fuente: elaboración propia

Al analizar esta pregunta en relación a la procedencia de las personas entrevistadas (Tabla 3) encontramos datos muy similares. Hay mayoría de personas (en los tres países de origen) que indicaron ver ambos tipos de televisión:

Tabla 3. Consumo de televisión (italiana, de otros países o ambas) según país de origen

\begin{tabular}{|c|c|c|c|c|}
\hline \multirow{2}{*}{ País de origen } & \multicolumn{3}{|c|}{ ¿Sueles ver la televisión italiana o la de otros países? } \\
\cline { 2 - 5 } & Ambas & $\begin{array}{c}\text { La televisión de } \\
\text { otros países }\end{array}$ & $\begin{array}{c}\text { La televisión } \\
\text { italiana }\end{array}$ & \multirow{2}{*}{ Total } \\
\hline China & $9(56,25 \%)$ & $1(6,25 \%)$ & $6(37,5 \%)$ & $16(100 \%)$ \\
\hline Filipinas & $10(55,55 \%)$ & & $8(44,44 \%)$ & $18(100 \%)$ \\
\hline Sri Lanka & $12(63,15)$ & & $7(36,84 \%)$ & $19(100 \%)$ \\
\hline Total & $31(58,49 \%)$ & $1(1,88 \%)$ & $21(39,62 \%)$ & $53(100 \%)$ \\
\hline
\end{tabular}

Fuente: elaboración propia

Con respecto a la formación de las personas entrevistadas y el tipo de televisión que normalmente ven (Tabla 4) destacamos, por un lado, el dato de que hay muchas más personas con un nivel medio-superior que indicaron ver ambos tipos de televisión con respecto a las que indicaron ver solo la televisión italiana con el mismo nivel formativo; y por otro que quienes tienen estudios universitarios y estudios medios-inferiores ven en casi en la misma medida ambas televisiones o solo la italiana:

Tabla 4. Consumo de televisión (italiana, de otros países o ambas) según nivel de formación

\begin{tabular}{|c|c|c|c|c|}
\hline \multirow{2}{*}{ Formación } & \multicolumn{2}{|c|}{ ¿Sueles ver la televisión italiana o la de otros países? } \\
\cline { 2 - 5 } & Ambas & $\begin{array}{c}\text { La televisión de } \\
\text { otros países }\end{array}$ & $\begin{array}{c}\text { La televisión } \\
\text { italiana }\end{array}$ & Total \\
\hline Doctorado/máster & $3(75 \%)$ & & $1(25 \%)$ & $4(100 \%)$ \\
\hline $\begin{array}{c}\text { Estudios } \\
\text { universitarios }\end{array}$ & $7(50 \%)$ & $1(7,14 \%)$ & $6(42,85 \%)$ & $14(100 \%)$ \\
\hline Media-superior & $18(64,28 \%)$ & & $10(35,71 \%)$ & $28(100 \%)$ \\
\hline Media-inferior & $3(42,85 \%)$ & & $4(57,14 \%)$ & $7(100 \%)$ \\
\hline $\begin{array}{c}\text { Estudios } \\
\text { elementales }\end{array}$ & & & & \\
\hline Total & $\mathbf{3 1 ( 5 8 , 4 9 \% )}$ & $\mathbf{1 ~ ( 1 , 8 8 \% )}$ & $\mathbf{2 1 ~ ( 3 9 , 6 2 \% )}$ & $\mathbf{5 3 ( 1 0 0 \% )}$ \\
\hline
\end{tabular}

Fuente: elaboración propia

RAE-IC, Revista de la Asociación Española de Investigación de la Comunicación vol. 8, núm. 15 (2021), 292-314 
Y por último, en relación al empleo (Tabla 5), ya comentamos en la descripción de la muestra que casi todas las personas entrevistadas se encontraban en situación activa, trabajando fuera del hogar. Entre las mismas la opción de respuesta mayoritaria fue la de quienes indicaron ver ambos tipos de televisión, frente a quienes manifestaron ver solo la italiana. Y entre quienes trabajaban en su propio hogar el número de respuestas de una y otra opción fue muy similar:

Tabla 5. Consumo de televisión (italiana, de otros países o ambas) según situación laboral

\begin{tabular}{|c|c|c|c|c|}
\hline \multirow{2}{*}{ Situación laboral } & \multicolumn{4}{|c|}{ ¿Sueles ver la televisión italiana o la de otros países? } \\
\cline { 2 - 5 } & Ambas & $\begin{array}{c}\text { La televisión de } \\
\text { otros países }\end{array}$ & $\begin{array}{c}\text { La televisión } \\
\text { italiana }\end{array}$ & Total \\
\hline Activo & $29(60,41 \%)$ & $1(2,08 \%)$ & $18(37,5 \%)$ & $48(100 \%)$ \\
\hline $\begin{array}{c}\text { Trabajo en el } \\
\text { propio domicilio }\end{array}$ & $2(40 \%)$ & & $3(60 \%)$ & $5(100 \%)$ \\
\hline Total & $\mathbf{3 1 ( 5 8 , 4 9 \% )}$ & $\mathbf{1 ~ ( 1 , 8 8 \% )}$ & $\mathbf{2 1 ( 3 9 , 6 2 \% )}$ & $\mathbf{5 3 ~ ( 1 0 0 \% )}$ \\
\hline
\end{tabular}

Fuente: elaboración propia

Los resultados de esta pregunta nos permiten reflexionar sobre los anclajes identitarios que la población migrante construye con respecto a sus lugares de residencia actual y con respecto a sus lugares de origen. Ello pensado a través del uso y consumo que hacen estas personas de la televisión: ¿es más frecuente que las personas que han migrado vean la televisión de sus países de origen? ¿la del país de residencia actual? ¿̇o ambas? Vemos cómo el porcentaje de personas que solo ven televisión "no italiana" es irrelevante en la muestra. Se trata de una sola mujer, menor de 40 años, de origen chino, con estudios universitarios y en activo. Podemos inferir con ello que existe entre las personas de la muestra considerada un deseo generalizado de conocer la sociedad de acogida a través de la televisión italiana (bien consumiendo programación exclusiva de misma, bien compartiendo tiempo entre la televisión italiana y la televisión de otros países). Siendo así, se encontró que la mayoría de las personas entrevistadas optaron por indicar que ven ambos tipos de televisión, por lo que es posible considerar que entre las personas procedentes de la migración persiste un interés por seguir vinculados a sus lugares de origen a través de la televisión, al tiempo que descubren, se familiarizan y amplían su conocimiento de la sociedad de acogida viendo la televisión italiana. Entre quienes se manifestaron en este sentido, personas que ven de manera indistinta ambos 
tipos de televisión, destacan -aunque con datos muy similares- hombres frente a mujeres, las personas procedentes de Sri Lanka, quienes tienen estudios mediossuperiores y quienes están en activo en términos laborales.

\subsubsection{La televisión como recurso para el aprendizaje de la lengua}

Para seguir indagando sobre las relaciones entre el uso de la televisión y las experiencias y percepciones sobre su potencialidad como herramienta para la integración, también preguntamos sobre las posibilidades de experimentar un mayor y mejor aprendizaje de la lengua italiana al ver la televisión italiana ${ }^{5}$, obteniendo casi un $80 \%$ de respuestas positivas $(79,7 \%)$ y un $20 \%$ de respuestas negativas ${ }^{6}$. Pero lo interesante de esta cuestión, que pone de manifiesto una opinión mayoritaria sobre las bondades de la televisión como medio de aprendizaje de la lengua, fue indagar en las respuestas que las personas dieron para argumentar por qué y/o con respecto a qué aspectos entendían que ver televisión italiana mejoraba la competencia lingüística. A este respecto, procedimos con la formulación de una pregunta abierta a partir de la que obtuvimos dos tipos de respuestas: por un lado las que indicaban qué aspectos del proceso de aprendizaje de la lengua había potenciado ver la televisión italiana; y por otro respuestas que especificaban qué tipo de programas y espacios televisivos les habían sido útiles especialmente. Con respecto al primer tipo de respuestas, sin duda, lo que más sobresale son narraciones sobre la "adquisición de vocabulario":

\section{Ejemplo 1.}

Puedo decir que sí, inicialmente miré la televisión para aprender algunas palabras más del idioma italiano. Miré la red local para obtener más información sobre la ciudad de Messina. La publicidad me ayudó. ${ }^{7}$ (Hombre de 49 años, originario de Filipinas, educación media-superior, laboralmente en activo. Mesina, 2019).

Ejemplo 2.

\footnotetext{
${ }^{5}$ Ha favorito la televisione italiana l'apprendimento della lingua italiana?

${ }^{6}$ En este caso hubo un valor perdido.

${ }^{7}$ Posso dire di si, inizialmente guardavo la televisione per imparare qualche vocabolo in più della lingua italiana. Guardavo la rete locale per avere più informazioni sulla città di Messina. La pubblicità mi ha aiutato.
} 
Ya sabía italiano pero aprendí muchos más términos. ${ }^{8}$ (Hombre de 41 años, originario de Sri Lanka, educación media-superior, laboralmente en activo. Mesina, 2019).

Ejemplo 3.

Vi dibujos animados cuando estaba en Prato y aprendí muchas palabras y modismos. ${ }^{9}$ (Hombre de 42 años, originario de China, educación media-superior, laboralmente en activo. Mesina, 2019).

Hasta un $38,3 \%$ de las personas que responden a esta cuestión se manifestaron en este sentido; la segunda respuesta más común $(23,4 \%)$ sobre este parecer fue indicar una mejora de la "comprensión auditiva":

\section{Ejemplo 4.}

A través de los programas he mejorado el idioma italiano... escuchando y repitiendo. ${ }^{10}$ (Mujer de 37 años, originaria de Sri Lanka, estudios universitarios, laboralmente en activo. Mesina, 2019).

\section{Ejemplo 5.}

Escuchando sobre todo los programas en los que se habla (talk shows) que tratan temas para debatir y hablar. ${ }^{11}$ (Mujer de 50 años, originaria de Sri Lanka, estudios universitarios, laboralmente en activo. Mesina, 2019).

\section{Ejemplo 6.}

Escuchar el diálogo en la televisión ayuda. Aunque he estudiado mucho el idioma italiano primero en China y luego en Italia. ${ }^{12}$ (Mujer de 34 años, originaria de China, estudios de doctorado/máster, laboralmente en activo. Mesina, 2019).

Y, por último, también se registraron algunas respuestas (el 10,6\%) que señalaron haber mejorado la pronunciación o incluso la gramática al ver la televisión italiana.

\footnotetext{
${ }^{8}$ Conoscevo già l'italiano ma ho imparato molti più termini.

${ }^{9}$ Vedevo cartoni quando ero a Prato e imparato tante parole e modi di dire.

${ }^{10}$ Attraverso i programmi ho migliorato lingua italiana....sentendo e ripetendo.

${ }^{11}$ Attraverso l'ascolto soprattutto dei programmi dove si parla (talk show) che trattano argomenti su cui discutere e parlare.

12 Sentire dialoghi in tv aiuta. Anche se io ho studiato molto la lingua italiana prima in Cina e poi in Italia.
} 
Con respecto al segundo tipo de respuestas, las que especificaban qué programas y espacios televisivos les habían sido útiles para la mejora del aprendizaje de la lengua, aunque menos frecuentes que las anteriores (el $27,7 \%$ de las respuestas incluían información de este tipo), hayamos datos interesantes. Esta clase de narraciones destacaban sobre todo la utilidad de la programación infantil, pero también de la publicidad, en el proceso de aprendizaje de la lengua:

\section{Ejemplo 7.}

Por ejemplo, inicialmente miré anuncios que tienen un lenguaje simple y fácil relacionado con imágenes o dibujos animados. ${ }^{13}$ (Mujer de 32 años, originaria de Filipinas, estudios universitarios, laboralmente en activo. Mesina, 2019).

\section{Ejemplo 8.}

Al principio, principalmente veía dibujos animados y comerciales para aprender palabras. ${ }^{14}$ (Mujer de 42 años, originaria de China, estudios medios-inferiores, ama de casa. Mesina, 2019).

Ambos tipos de programación porque se caracterizan por un lenguaje sencillo.

Con esta pregunta pudimos ver cómo las personas participantes en la investigación desplegaban sus propias estrategias y recursos para aprovechar al máximo algunas de las particularidades de cierto tipo de programación. El lenguaje accesible de los programas infantiles es un ejemplo de ello; pero también la publicidad que -con sus mensajes cortos y sencillos- facilita la adquisición de vocabulario frente a otro tipo comunicación más densa.

\subsubsection{La televisión como herramienta para conocer la cultura}

Finalmente preguntamos, otra vez con una pregunta de carácter abierto, por el consumo televisivo como posible recurso para conocer sobre la cultura y costumbres italianas ${ }^{15}$. Sobre esta cuestión la mayoría de las personas, de nuevo, respondieron ${ }^{16}$ de

\footnotetext{
${ }^{13}$ Per esempio, inizialmente guardavo le pubblicità che hanno un linguaggio semplice e facile legato alle immagini o i cartoni animati.

${ }^{14}$ All'inizio guardavo soprattutto cartoni animati e pubblicità per imparare le parole.

${ }^{15}$ Attraverso i programmi televisivi hai conosciuto usi, abitudini e costumi degli italiani?

${ }^{16}$ Registramos un valor perdido.
} 
manera positiva (un $66,1 \%$ de la muestra), aunque lo hicieron en menor medida que cuando se les preguntó por la lengua (recordemos, casi un $80 \%$ afirmó que ver la televisión italiana contribuía al aprendizaje de la lengua). A continuación pedimos a los/as entrevistados/as que, si habían respondido positivamente, desarrollasen sus argumentos con algunos ejemplos ${ }^{17}$. Las respuestas de los/as entrevistados/as a este respecto hablaban sobre todo (un $39,5 \%$ de las mismas) del conocimiento de tradiciones y fiestas a través de la televisión:

Ejemplo 9.

Por ejemplo, las tradiciones que existen en la ciudad de Messina, a través del RTP (canal de Messina). Cuando llegué vi esos programas en los que se contaban ciudades italianas. ${ }^{18}$ (Hombre de 49 años, originario de Filipinas, estudios medios-superiores, laboralmente en activo. Mesina, 2019).

En segundo lugar, un $34,9 \%$ de las respuestas, indicaban que a través de televisión se aprendía sobre la cocina italiana:

Ejemplo 10.

Por ejemplo, aprendí a cocinar. Viendo La prova del cuoco aprendí a hacer lasaña. ${ }^{19}$ (Mujer de 50 años, originaria de Sri Lanka, estudios universitarios, laboralmente en activo. Mesina, 2019).

Aunque la mayoría de las respuestas en esta línea señalaban otras tantas cuestiones relacionadas con la gastronomía: tradiciones, costumbres, fiestas, lugares y zonas de Italia, etc.:

Ejemplo 11.

Sí, comidas italianas, también de los hábitos de los italianos en algunas fiestas, mirando La prova del cuoco por ejemplo, aprendía cocinar comidas típicas de Navidad o Semana

\footnotetext{
17 Puoi raccontarci qualche esempio?

${ }_{18}$ Per esempio, le tradizioni che ci sono nella città di Messina, attraverso la RTP (canale di Messina). Quando sono arrivato guardavo quei programmi in cui si raccontavano le città italiane.

${ }^{19}$ Ad esempio, ho imparato a cucinare. Guardando La prova del cuoco ho imparato a fare le lasagne.
} 
Santa; Viendo la serie vi muchos lugares italianos y muchas cosas hermosas que ver. ${ }^{20}$ (Mujer de 31 años, originaria de Sri Lanka, estudios medios-superiores, laboralmente en activo. Mesina, 2019).

$Y$, en tercer lugar, vimos cómo entre las respuestas también destacaban aquellas que indicaban que a través de la televisión se pudo tener conocimiento de las formas de ser y la forma de vida de los/as italianos/as. Un $23,3 \%$ de las respuestas apuntaban esta cuestión:

\section{Ejemplo 12.}

He aprendido a comprender muchas cosas como funcionan en Italia. Las diferencias también en la forma de vida entre el norte y el sur de Italia. ${ }^{21}$ (Hombre de 36 años, originario de Filipinas, estudios medios-superiores, laboralmente en activo. Mesina, 2019).

Aparecieron otros aspectos relacionados con la cultura, como la historia y la geografía del país, el arte o la moda, aunque en menor medida que los anteriores.

Es interesante observar cómo en la mayoría de las ocasiones las personas entrevistadas indicaron que aprendían sobre todas estas cuestiones viendo un tipo de programación específica:

\section{Ejemplo 13.}

Sí, a veces también veo retransmisiones donde preparan platos regionales. Por ejemplo, en "Ricette all'italiana", además de hablar de recetas, también se cuentan curiosidades de algunos lugares. ${ }^{22}$ (Mujer de 48 años, originaria de Filipinas, estudios mediossuperiores, ama de casa. Mesina, 2019).

Ejemplo 14.

\footnotetext{
${ }^{20} \mathrm{Si}$, i cibi italiani, anche delle abitudini degli italiani in alcune feste.Guardando la prova del cuoco per esempio, ho imparato a cucinare cibi tipici del Natale o della Pasqua; guardando delle serie ho visto tanti posti italiani e tante cose belle da vedere. ${ }^{21} \mathrm{Ho}$ imparato a capire tante cose come funzionano in Italia. Le differenze anche di modo di vita tra nord e sud Italia.

${ }^{22} \mathrm{Si}$, a volte guardo anche trasmissioni dove preparano piatti di regione. Per esempio, a "Ricette all'italiana" oltre a parlare di ricette, sono raccontate anche curiosità di alcuni posti.
} 
Muchas tradiciones de la ciudad donde vivo, las conocí con programas de televisión locales. ${ }^{23}$ (Mujer de 34 años, originaria de Filipinas, estudios medios-superiores, ama de casa. Mesina, 2019).

En concreto programas sobre cocina y canales de la televisión local.

Los resultados de esta pregunta nos permitieron conocer las preferencias de las personas entrevistadas en cuanto al contenido televisivo afín a sus intereses y necesidades como personas procedentes de las migraciones y que -por eso mismo- no conocen o no están familiarizadas aún con los códigos culturales de la sociedad de acogida. Siendo así vimos cómo la proximidad que aporta la televisión local es un valor añadido y muy tenido en cuenta por estas personas, dado que les permite experimentar en su día a día aquello que están conociendo a través de una pantalla. Así mismo fue interesante observar cómo los programas gastronómicos, al abordar aspectos que van más allá "de la cocina", son usados por las personas migrantes para conocer y familiarizarse con muchos otros componentes de la cultura (costumbres, fiestas, tradiciones, hábitos, formas de ser y de estar, etc.).

\section{DISCUSIÓN Y CONCLUSIONES}

En este trabajo hemos analizado el uso que hace de la televisión una muestra de personas migrantes de origen asiático en la ciudad de Mesina (Italia), con el objetivo de conocer las posibilidades de la misma como herramienta de integración social. Hemos focalizado para ello nuestro interés en conocer qué tipo de televisión es la más vista por las personas migrantes participantes en la investigación; conocer las experiencias de uso de este medio de comunicación en tanto que recurso para la capacitación lingüística; y, en tercer lugar, conocer las opiniones sobre los aportes de la televisión como recurso para familiarizarse con aspectos culturales de Italia.

Con este estudio de caso se ha podido constatar la importancia de la televisión en tanto que medio de comunicación (aún) central en la vida cotidiana de las personas procedentes de la inmigración. Los resultados de la entrevista indican que casi la

${ }^{23}$ Molte tradizioni della città in cui abito, ho conosciuto con programmi tv locali. 
práctica totalidad de las personas ven la televisión italiana o al mismo tiempo esta y la televisión de sus países de origen. Es ínfimo el porcentaje de personas que solo consume contenidos televisivos de su país de origen. Esta cuestión es un primer elemento de reflexión sobre las posibles bondades percibidas en consumir al mismo tiempo un tipo y otro de televisión; y de no anclarse a través de los medios de comunicación en las sociedades de origen. Cuando hemos preguntado por el aprendizaje de la lengua o mejora en la competencia de misma y el conocimiento de los patrones y características culturales del país de acogida a partir del uso de la televisión, hemos visto cómo las personas entrevistadas han manifestado mayoritariamente una opinión muy favorable al respecto. Las narraciones obtenidas nos hablan en primera persona de adquisición de un mayor vocabulario, de familiarización con las formas de hablar, del conocimiento de tradiciones y folclore, del aprendizaje de hábitos y costumbres, etc. La teoría de usos y gratificaciones nos habla a este respecto de que, justamente, un tipo de gratificaciones que las personas pueden obtener de los medios tiene que ver con estos aspectos: obtener información/conocimiento susceptible de ser empleado para mejorar las interacciones sociales (Katz et al., 1974; Livolsi, 2020). Es por ello que hemos de concluir que, inevitablemente, la televisión está contribuyendo a la integración social de estas personas; o dicho de otra forma: las personas procedentes de las migraciones aprovechan -se gratifican de- ciertos contenidos y formatos que proporciona la televisión para reforzar el aprendizaje de la lengua y el conocimiento de la sociedad italiana (elementos esenciales dentro de los procesos de integración). No olvidemos, sin embargo, que la integración es más un "proceso" que un "estado", y que por eso mismo puede ser de ida y vuelta, y en el mismo están implicadas todos los componentes de una sociedad, no solo las personas migrantes (Olmos Alcaraz, 2009, 2020; Olmos Alcaraz y Lastres, 2018).

Los aportes de los que hablamos se observan de forma más clara, si se quiere, en el caso de la lengua. Aunque también es visible -en las respuestas obtenidas-que la televisión es usada para familiarizarse con algunos elementos constitutivos de la cultura italiana. No podemos sin embargo, a partir de esta idea, caer en el reduccionismo de pensar que se "puede aprender la cultura". Ello supondría entender la misma de forma cosificada, 
esencializada y en folclorizada (Díaz de Rada, 2010). Es por ello que, a pesar de reconocer el potencial del medio televisivo como herramienta para la integración, no es posible concluir con argumentos superficiales y simplistas al respecto. Indudablemente en ese "aprendizaje" narrado por las personas entrevistadas también hay un aprendizaje de estereotipos y prejuicios sobre "la cultura italiana", sin que ello reste valor al uso pueda hacerse de este medio; y sin que ello reste valor al potencial integrador de los medios de comunicación en general (Lorite, 2004a, 2004b; Van Dijk, 1997, 2009), y de la televisión en particular (Navarro, 2015). Proponemos seguir trabajando en esta línea, en futuros trabajos, para conocer sobre representaciones sociales sobre la integración social-pero también de la exclusión, de la desigualdad- en los medios de comunicación, en particular la televisión; y cómo ello es experimentado y pensado por las propias personas migrantes.

\section{REFERENCIAS BIBLIOGRÁFICAS}

Altarriba, M. (2008). Usos i actituds dels immigrants davant dels mitjans de comunicación. Barcelona: Consell de l'Audiovisual de Catalunya (CAC).

Balma, C. (2001). Colori della realtà. Immagini dell'immigrazione nei programmi di servizio RAI. II nuovo spettatore, 5, 30-41.

Bañón, A. (2004). La representación periodística de los inmigrantes y la enseñanza del análisis crítico del discurso. Glosas didácticas, 12, 127-142.

Bañón, A. (2007). El discurso periodístico a propósito del viaje de los inmigrantes pobres. En R. Zapata-Barrero y T. van Dijk (Eds.), Discursos sobre la inmigración en España. Los medios de comunicación, los parlamentos y las administraciones, 45-68. Barcelona: Fundación CIDOB.

Belluatl, M. (2015). Dalla fiction ai TG: la TV che piace agli immigrati. Problemi dell'informazione, $X L, 1,245-248$.

Binotto, M., Bruno, M. y Lai, V. (2016). Tracciare confini. L'immigrazione nei media italiani. Milán: Franco Angeli. 
Calvanese, E. (2011). Media e immigrazione tra stereotipi e pregiudizi. La rappresentazione dello straniero nel racconto giornalistico. Milán: Franco Angeli.

Caritas (2012). Dossier Statistico Immigrazione. $22^{\circ}$ Rapporto. Roma: Edizioni IDOS.

Cava, G. (2019). Breve historia de la televisión italiana destinada a inmigrantes: el caso de Babel TV. Index.comunicación, 9(3), 59-74. Recuperado de https://journals.sfu.ca/indexcomunicacion/index.php/indexcomunicacion/article/view $\not 561$.

Chavero, H. y García, N. (2005). Los hábitos del consumo televisivo de una comunidad extranjera: el caso de estudiantes latinoamericanos en Barcelona. Zer-Revista de estudios de comunicación, 19, 191-204.

Díaz de Rada, A. (2010). Cultura, Antropología y otras tonterías. Madrid: Trotta.

García, F. J., Granados, A., Olmos Alcaraz, A. y Martínez, R. (2014). Cuando no hablamos de integración: análisis de la imagen del alumnado "inmigrante" en la prensa en España. Estudios Sobre El Mensaje Periodístico, 20(2), 1081-1101. https://doi.org/10.5209/rev_ESMP.2014.v20.n2.47051

González, M. E. (2009). La consolidación de las publicaciones para extranjeros en España. Revista Latina de Comunicación Social, 64, 708-724. https://doi.org/10.4185/RLCS-64-2009-856-708-724

Granados, A. (1998). La imagen del inmigrante extranjero en la en la prensa española: ABC, Diario 16, El Mundo y El País (1985-1992). Tesis doctoral. Granada: Universidad de Granada.

Granados, A. (2013). Las representaciones de las migraciones en los medios de comunicación. Madrid: Trotta.

Igartua, J.J., Ortega-Mohedano, F. y Arcila-Calderón, C. (2020). The uses of communication in the time of coronavirus. A cross-cultural study. El profesional de la información, 29(3), e290318. https://doi.org/10.3145/epi.2020.may.18 
Katz, E., Blumler J., Gurevitch, M. (1974). Utilization of Mass Communication by the Individual. En J. Blumler y E. Katz (Eds.), The Uses of Mass Communication: Current Perspectives on Gratifications Research (pp. 19-31). Beverly Hills: Sage Publications.

Kaya, A. (2017). Populismo e inmigración en la Unión Europea. Anuario CIDOB de la Inmigración, [en línea], 2017, 52-79.

https://doi.org/10.24241/AnuarioCIDOBInmi.2017.52

King, R. y Wood, N. (2013). Media and migration. Constructions of Mobility and Difference. Londres y Nueva York: Routledge.

Livolsi, M. (2020). Manuale di sociologia della comunicazione. Roma: Laterza.

Lorite, N. (2004a). Tratamiento de la inmigración en España: 2002. Madrid: MTAS.

Lorite, N. (2004b). Como tratar la inmigración en los medios pensando en la inteculturalidad. Red digital: Revista de Tecnologías de la Información y Comunicación Educativas, 5, s/p.

Lorite, N. (2011). Cómo representa la televisión informativa a la inmigración subsahariana y transmite estereotipos negativos. En G. Franco y D. García (Coord.), Mujer, educación y migraciones en África (pp. 61-80). Madrid: La Catarata.

Navarro, L. (2014). Medios de comunicación creados por inmigrantes marroquíes en España: entre la movilización social y el control político. Commons: revista de comunicación y ciudadanía digital, 3(2), 78-115

Navarro, L. (2015). Televisión pública y diversidad cultural en España: el caso de los programas dirigidos a «inmigrantes». Migraciones, 38, 111-135.

Meli, A. (2011). Le tante voci della nuova Italia multiculturale. Libertà civil, 2, 29-35. Martínez, M. (2013). Explorando la construcción discursiva de las mujeres y los menores inmigrantes en una muestra de noticias de radio. Tonos Digital, 25, 1-14.

Martínez, M. (2015). Apuntes sobre las mujeres inmigrantes en la prensa española: deconstruyendo la invisibilidad. En G. Esteban de la Rosa y A. Tandian (Coord.), Mejora 
de las condiciones de la emigración de las personas del África sudsahariana a la Unión Europea (pp.173-184). Granada: Comares.

Martínez, M. y Olmos Alcaraz, A. (2015). Menores y mujeres inmigrantes en la radio y la televisión públicas: imágenes sesgadas y ficciones mediáticas. Revista Tonos Digital, Recuperado de http://www.tonosdigital.es/ojs/index.php/tonos/article/view/1322

Matsaganis, M., Katz, V. y Ball, S. (2011). Understanding Ethnic Media. Producers, Consumers and Societies. Thousand Oaks. Thousand Oaks, CA: Sage.

Musarò, M. y Parmiggiani, P. (2014). Media e migrazioni. Milán: Franco Angeli.

Olmos Alcaraz, A. (2009). La población inmigrante extranjera y la construcción de la diferencia. Discursos de alteridad en el sistema educativo andaluz. Universidad de Granada: Granada.

Olmos Alcaraz, A. (2013). "Pateras, embarazadas y prostitución": representaciones y discursos sobre la mujer inmigrante en la televisión española. Fonseca Journal of Communication, 7, 72-99. Recuperado de https://revistas.usal.es/index.php/21729077/article/view/11706

Olmos Alcaraz, A. y Lastres, N. (2018). Transitando por los bordes de la integración: una aproximación etnográfica a políticas educativas y experiencias escolares de alumnado que desconoce la lengua vehicular de la escuela en Andalucía (España). Gazeta de Antropología, 34(1), 1-28. Recuperado de http://www.gazetaantropologia.es $/ \mathrm{p}=5064$

Olmos-Alcaraz, A. (2020). Análisis de algunos vectores sobre las migraciones internacionales en España: las políticas de integración e integración educativa a debate. Revista Política, Globalidad y Ciudadanía, 6(11), 203-223. https://doi.org/10.29105/pgc6.11-9

Osmani, X. (2019). Discurso político sobre la inimgración. Análisis comparativo entre Grecia, España e Italia. Tesis doctoral. Madrid: Universidad Carlos III de Madrid. 
Retis, J. (2004). La imagen del otro: inmigrantes latinoamericanos en la prensa nacional española. Sphera Pública, 4, 119-140.

Retis, J. (2013). Immigrant latina images in mainstream media: class, race and gender in public discourse in the United States and Spain. En M. Martinez (Ed.), Discourses on Immigration in Times of Economic Crisis: A Critical Perspective (pp. 29-59). Newcastle: Cambridge Scholars Publishing.

Retis, J. (2014). ¿Consumidores o ciudadanos?: Prácticas de consumo cultural de los inmigrantes latinoamericanos en España. Fronteras, 1(1), 2014, 71-96.

Retis, J. (2018). Inmigrantes latinoamericanos en ciudades globales: aproximaciones interdisciplinarias en el análisis de las prácticas comunicativas, mediáticas y culturales. Contratexto: revista de la Facultad de Comunicación de la Universidad de Lima, 30, 2018, 20-40. https://doi.org/10.26439/contratexto2018.n030.3147

Rosell, M. M. (2008). La prensa para inmigrantes se profesionaliza. Cuadernos de periodistas: revista de la Asociación de la Prensa de Madrid, 13, 81-90.

Salanitro, A. (2012). L'immigrazione a Messina. Ricerca sugli stranieri residenti. Messina: Anolf-Cisl.

Santillán, J. R. (2008). Medios latinos en España. Razón y palabra, 63. Recuperado de http://www.razonypalabra.org.mx/n63/varia/rsantillan.html

Van Dijk, T. (1997). Racismo y análisis crítico de los medios. Barcelona: Paidós.

Van Dijk, T. (2009). Racismo y poder. Barcelona: Gedisa. 